\title{
Universal properties of the ultracold Fermi gas
}

\author{
Shizhong Zhang and Anthony J. Leggett \\ Department of Physics, University of Illinois at Urbana-Champaign, 1110 West Green Street, Urbana, Illinois 61801-3080, USA
}

(Received 10 September 2008; revised manuscript received 18 November 2008; published 2 February 2009)

\begin{abstract}
We present some general considerations on the properties of a two-component ultracold Fermi gas along the BEC-BCS crossover. It is shown that the interaction energy and the free energy can be written in terms of a single dimensionless function $h(\xi, \tau)$, where $\xi=-\left(k_{F} a_{S}\right)^{-1}$ and $\tau=T / T_{F}$. The function $h(\xi, \tau)$ incorporates all the many-body physics and naturally occurs in other physical quantities as well. In particular, we show that the average rf-spectroscopy shift $\overline{\delta \omega}(\xi, \tau)$ and the molecular fraction $f_{c}(\xi, \tau)$ in the closed channel can be expressed in terms of $h(\xi, \tau)$ and thus have identical temperature dependence. The conclusions should have testable consequences in future experiments.
\end{abstract}

DOI: 10.1103/PhysRevA.79.023601

PACS number(s): 03.75.Ss, 03.75.Hh, 74.20.Fg

\section{INTRODUCTION}

Over the past few decades, there has been considerable effort and progress in understanding the physics of ultracold Fermi gases [1-4]. In general, the theoretical investigations fall into two categories depending on how one incorporates the physics of Feshbach resonances. In the so-called singlechannel model, one neglects the closed-channel component, while incorporating its effects through the open-channel scattering length $a_{s}$, given by

$$
a_{s}(B)=a_{\mathrm{bg}}\left(1-\frac{\Delta B}{B-B_{0}}\right),
$$

where $a_{\mathrm{bg}}$ is the background scattering length in the absence of inter-channel coupling and $B$ is the external magnetic field. $\Delta B$ is the width of the resonance and $B_{0}$ is the position of the resonance. See Table V in Ref. [5] for a list of values of these parameters for the alkali-metal elements currently under investigation. The approximation is valid in the case of a so-called broad resonance where $\epsilon_{F} \ll \delta_{c}$. Here $\epsilon_{F}$ is the Fermi energy of the system and $\delta_{c}=\frac{(\Delta \mu \Delta B)^{2}}{2 \hbar^{2} / m a_{b g}^{2}}$ [6], characterizing the typical energy scale associated with the two-body Feshbach resonance [7]. $\Delta \mu$ is the magnetic moment difference between open and closed channels. The physics of the single-channel model is essentially the same as in the crossover model studied decades ago in the literature [8-10]. Most experimental systems (e.g., ${ }^{6} \mathrm{Li}$ at magnetic field $B$ $=834 \mathrm{G})$ fall into this category. In general, any system with sufficiently low density will be described by a single-channel model.

The problem associated with a single-channel model is easily stated: Given a spin- $\frac{1}{2}$ Fermi gas with both spin components equally populated, with interactions only between opposite spin states, characterized by the $s$-wave scattering length $a_{s}(B)$, what are the ground state and thermodynamic properties? At zero temperature, neglecting finite range corrections, the only relevant parameter is $\xi=-\left(k_{F} a_{s}\right)^{-1}$. The basic question concerning the static properties of the system is how to find the ground-state energy $E(\xi)$. At finite temperature, the most important questions are the calculation of the thermodynamic potential and in particular the location of the phase transition boundary $T_{C}(\xi)$ as a function of $\xi$; see Ref. [11] and references therein. Up to now, these problems have not been amenable to analytic solutions; our most reliable knowledge of those quantities comes from Monte Carlo simulations [12-18].

For most experiments, it is sufficient to use the singlechannel model as the theoretical framework to interpret them. However, there are certain cases in which we are interested in the physics of the closed channel specifically for a broad Feshbach resonance, as in the case of the Rice experiment to be discussed later [19]. Furthermore, in the so-called narrow resonance case in which $\epsilon_{F} \geqslant \delta_{c}$, the molecular states in the closed channel cannot be neglected. For these reasons, we have to start with the more general two-channel model [20-22], which incorporates the closed channel on the same footing as the open channel. Typically, one introduces a bosonic operator $\Psi(\vec{r})$, which creates a closed-channel molecule with center-of-mass position $\vec{r}$ and incorporates its effects through the coupling term in the Hamiltonian,

$$
\widetilde{g}\left(\Psi^{\dagger}(\vec{r}) \psi_{\uparrow}(\vec{r}) \psi_{\downarrow}(\vec{r})+\text { H.c. }\right),
$$

where $\widetilde{g}$ is the bare coupling constant. Note that the coupling scheme above enforces the momentum conservation in the conversion processes and thus the momentum distribution of closed-channel molecules is intimately connected with the open-channel pair states. We shall return to this point later. Note also that the internal structure of the closed-channel molecule is frozen as a result of its high internal excitation energy, which is much larger than any other energy scales relevant for many-body physics.

In general, exact solutions or even general statements about the above two models are quite difficult. However, at resonance, i.e., $\xi=0, a_{s}$ drops out of the problem and we are left with only two energy scales, $\epsilon_{F}=\hbar^{2} / 2 m\left(3 \pi^{2} n\right)^{2 / 3}$ and the temperature $T$. Here $m$ is the mass of the atom and $n$ is the density of the system. Thus, it follows from dimensional analysis that one may write the average single-particle energy of the system at finite temperature as $\epsilon(\xi=0, \tau)$ $=\epsilon_{F} f_{E}(\xi=0, \tau)$, where $f_{E}(\xi=0, \tau)$ is a dimensionless function and $\tau=T / T_{F} \equiv k_{B} T / \epsilon_{F}$, where $k_{B}$ is the Boltzmann constant. In particular, at $\tau=0$, the average single-particle energy is proportional to $\epsilon_{F}$, with universal constant $f_{E}(\xi=0, \tau=0)$ $\equiv \frac{3}{5}(1+\beta)$. The parameter $\beta$ has been calculated in many ways in the literature and is in good agreement with experi- 
ments. See Table II in [2] for a summary of values of $\beta$ obtained theoretically and experimentally. By the same argument, one can write down other thermodynamic quantities of the system, with the conclusion that the thermodynamic properties of the system are universal regardless of the particular system under investigation [23].

The universal thermodynamics works only at unitarity. However, it is possible to generalize the idea to the parameter space where $\xi \neq 0$. It is recognized that for $d>2$, the system is controlled by an unstable fixed point that resides near the Feshbach resonance in the zero density limit with attractive interactions [24]. By utilizing a large- $N$ expansion as applied to the $\operatorname{Sp}(2 N)$ model [24,25], one can calculate the scaling form of the canonical free energy of the system. In Ref. [25], the same technique has been used to investigate the polarized case as well. The $\epsilon$ expansion has also been used to investigate the properties of the two-component Fermi gas away from resonance [26,27].

There is, however, another form of "universality" that is more deeply rooted in the actual physical properties of the system. For example, by examining the short-range form of the many-body wave function, one can show that several physical quantities (interaction energy, rf-spectroscopy shift, etc.) depend on temperature $\tau$ through one universal function $h(\xi, \tau)$. This universal dependence comes about because of one peculiar property of the dilute Fermi gases: the range of the interaction is much smaller than the interparticle distance, i.e., $k_{F} r_{0} \ll 1$, where $r_{0}$ is the range of the potential. Thus, important effects associated with interactions come mostly from two-body encounters. The argument presented below can then be regarded as an expansion in terms of $k_{F} r_{0}$. Let us note that this argument can be trivially modified in the case of the imbalanced Fermi gas.

The organization of the paper is the following. In Sec. II, we give a general discussion of the physical system in terms of the two-body density matrix and separate the two-body and many-body contributions in it. In Sec. III, we apply the result of Sec. II to several physical quantities and show that they can be written in terms of one universal function $h(\xi, \tau)$ which carries all the many-body dependence. There are residual $\xi$ dependences as a result of the two-body physics, which can in principle be calculated without any reference to the many-body system. The temperature dependence of those physical quantities is universal and has experimental consequences as described in Sec. III. In IV, the main conclusions of the paper are summarized and discussed. In the Appendix, we give an alternative derivation of the linear $\xi$ dependence of the energy of the system away from resonance, based on the many-body wave functions.

\section{GENERAL SETUP}

The difficulties involved in analyzing either the singlechannel or the two-channel model are often expressed as a lack of a small parameter because of the resonant interaction condition $n a_{s}^{3} \gg 1$, which prevents a relatively straightforward perturbation calculation as in the classic dilute Fermi gas [28-30]. The challenge lies in the correct implementation of the two-body physics, characterized by the "danger- ous" diverging scattering length $a_{s}$, into the many-body calculations. One way to circumvent the difficulty is to devise another small parameter, as in the $\epsilon$ expansion [26] or $1 / N$ expansion $[24,25]$ utilized in recent work. On the other hand, even though the parameter $n a_{s}^{3} \gg 1$, we still have the small parameter $k_{F} r_{0}$, where $r_{0}$ is the range of the potential. Note that in most investigations using the single- or two-channel model, the zero-range limit has already been taken; an exception is the case of a narrow resonance [31]. In the following, we will try to set up an approximation scheme that utilizes the smallness of $k_{F} r_{0}$. Even though it does not yield immediately a computational tool for the values of specific constants, say $\beta$, it does lead to some general conclusions independent of the approximation scheme employed in a specific investigation.

To motivate our discussion, let us consider the many-body wave function for a spin- $\frac{1}{2}$ Fermi system with both spin components equally populated. We denote the total number of atoms $N$. For example, we consider a collection of ${ }^{6} \mathrm{Li}$ atoms in their lowest two hyperfine-Zeeman states $(|1\rangle$ and $|2\rangle)$. Let us write down its many-body wave function as $\Psi\left(\vec{r}_{1} \sigma_{1}, \vec{r}_{2} \sigma_{2} \cdots \vec{r}_{N} \sigma_{N}\right)$. Now, we separate two atoms, say atom 1 and atom 2, with opposite spin orientations, far (compared to $\left.r_{0}\right)$ from all the other atoms $(3,4, \ldots, N)$, and ask what is the form of the many-body wave function when the distance between $\vec{r}_{1}$ and $\vec{r}_{2}$ is taken to lie within the range of two-body interacting potential, i.e., $\left|\vec{r}_{1}-\vec{r}_{2}\right| \lesssim r_{0}$. Since all the other $N-2$ atoms cease to interact with atom 1 and atom 2 , we conclude that

$$
\begin{aligned}
& \lim _{\left|\vec{r}_{1}-\vec{r}_{2}\right| \leq r_{0}} \Psi\left(\vec{r}_{1} \sigma_{1}, \vec{r}_{2} \sigma_{2} \cdots \vec{r}_{N} \sigma_{N}\right) \\
& \quad \propto \mathcal{A} \phi\left(\vec{r}_{1}-\vec{r}_{2}\right) \Sigma_{12} \Psi^{\prime}\left(\vec{r}_{3} \sigma_{3}, \vec{r}_{4} \sigma_{4} \cdots \vec{r}_{N} \sigma_{N}\right) .
\end{aligned}
$$

Here $\mathcal{A}$ is the trivial antisymmetrization operator and $\Sigma_{12}$ is the spin wave function of atoms 1 and 2. $\phi\left(\vec{r}_{1}-\vec{r}_{2}\right)$ is determined by the two-body interaction potential in the range $\left|\vec{r}_{1}-\vec{r}_{2}\right| \lesssim r_{0}$. In order for the other $N-2$ atoms to affect the form of $\phi\left(\vec{r}_{1}-\vec{r}_{2}\right)$, it is necessary that a third atom is at a distance $\lesssim r_{0}$. Such a process is highly unlikely for two reasons. First, the phase space of such an event is down by at least a factor $\left(k_{F} r_{0}\right)^{3}$ as compared with the two-body encounters. Secondly, in a spin- $\frac{1}{2}$ system, two of the three atoms close together must have the same spin, thus the Pauli exclusion principle will prevent such a process from occurring. In fact, to the extent that we can work entirely in terms of the $s$-wave scattering length $a_{s}$, we have automatically neglected the contributions from higher partial wave scattering, which are of order $\left(k_{F} r_{0}\right)^{2}$ or higher. We thus conclude that, to order $k_{F} r_{0}$, the short-range behavior of the many-body wave function (in particular its nodal structure) is determined by twobody physics. An important question pertains to the form of the two-body wave function $\phi\left(\vec{r}_{1}-\vec{r}_{2}\right)$, since, in general, the two-body potential can host several bound states. Here we note that since the many-body energy scale is much smaller than the energy splitting between the different energy levels in the potential well, it is easy to convince oneself that only the bound state that is closest to the scattering continuum is relevant. This state is merely the molecular state on the 
Bose-Einstein Condensate (BEC) side of the crossover. Here we emphasize that even on the BCS side, where the twobody bound state emerges above the scattering continuum, the above conclusion still holds. To conclude this intuitive discussion, we must point out that at this stage, we do not yet know the normalization of the short-range part of the manybody wave function. This will be determined by the manybody physics.

To make the above argument more precise, let us consider the two-body density matrix for a generic many-body system. The definition of the two-body density matrix is given by [7]

$$
\rho\left(\vec{r}_{1} \alpha, \vec{r}_{2} \beta ; \vec{r}_{3} \gamma, \vec{r}_{4} \delta ; t\right)=\left\langle\psi_{\alpha}^{\dagger}\left(\vec{r}_{1}, t\right) \psi_{\beta}^{\dagger}\left(\vec{r}_{2}, t\right) \psi_{\gamma}\left(\vec{r}_{3}, t\right) \psi_{\delta}\left(\vec{r}_{4}, t\right)\right\rangle .
$$

Here $\psi_{\alpha}\left(\vec{r}_{1}, t\right)$ is the Heisenberg field operator for a fermion with spin $\alpha$. In the following, we shall consider only an equilibrium situation and thus drop the time $t$ from the above expression. By the hermiticity property of the density matrix, we can decompose the two-body density matrix in the following form [7]:

$$
\rho\left(\vec{r}_{1} \alpha, \vec{r}_{2} \beta ; \vec{r}_{3} \gamma, \vec{r}_{4} \delta\right)=\sum_{i} n_{i} \phi_{\beta \alpha}^{(i) *}\left(\vec{r}_{2}, \vec{r}_{1}\right) \phi_{\gamma \delta}^{(i)}\left(\vec{r}_{3}, \vec{r}_{4}\right) .
$$

The eigenvalues $n_{i}$ and eigenfunctions $\phi_{\alpha \beta}^{(i)}\left(\vec{r}_{1}, \vec{r}_{2}\right)$ satisfy the following conditions: $\sum_{i} n_{i}=N(N-1)$ and $\Sigma_{\alpha \beta} \int d^{3} \vec{r}_{1} \int d^{3} \vec{r}_{2} \phi_{\alpha \beta}^{(i) *}\left(\vec{r}_{2}, \vec{r}_{1}\right) \phi_{\beta \alpha}^{(j)}\left(\vec{r}_{1}, \vec{r}_{2}\right)=\delta_{i j}$. As discussed above, in the case of a dilute Fermi gas, the only relevant parameter is $\xi=-\left(k_{F} a_{s}\right)^{-1}$ and $\tau$. Thus $n_{i}$ and $\phi_{\beta \alpha}\left(\vec{r}_{2}, \vec{r}_{1}\right)$ will depend on $\xi$ and $\tau$ parametrically. Now, by the argument given above in terms of the many-body wave function, we see that the short-range form of $\phi_{\alpha \beta}\left(\vec{r}_{1}-\vec{r}_{2}\right)$ will be determined by two-body physics, while many-body physics will determine the eigenvalues $n_{i}$ and the long-range part of the eigenfunctions. Our philosophy in the following will be to express several physical quantities in terms of the two-body density matrix and use the above facts to extract their universal dependence on temperature. To be successful, we need our expressions to pick up only the short-range part of the two-body density matrix so that all the temperature dependence will be carried by $n_{i}$ 's and the normalizations for the pair wave functions. Physically, as we change the temperature and the interaction strength, the occupation numbers of the pair wave functions $\phi_{\alpha \beta}^{(i)}$ change while the short-range part of the pair wave function remains the same. Let us thus consider an arbitrary short-range $\left(\sim r_{0}\right)$ function $s\left(\vec{r}_{1}-\vec{r}_{2}\right)$ and consider the integral

$$
\begin{aligned}
& \int d \vec{r}_{1} d \vec{r}_{2} s\left(\vec{r}_{1}-\vec{r}_{2}\right)\left\langle\psi_{1}^{\dagger}\left(\vec{r}_{1}\right) \psi_{2}^{\dagger}\left(\vec{r}_{2}\right) \psi_{2}\left(\vec{r}_{2}\right) \psi_{1}\left(\vec{r}_{1}\right)\right\rangle \\
& =\sum_{i} n_{i}(\xi, \tau) \int s\left(\vec{r}_{1}-\vec{r}_{2}\right)\left|\phi_{12}^{(i)}\left(\vec{r}_{1}, \vec{r}_{2}\right)\right|^{2} d \vec{r}_{1} d \vec{r}_{2} .
\end{aligned}
$$

It is clear that in the above equation, we need only retain the $s$-wave part of the pair wave function, since higher partial waves have vanishing probability at the origin and thus hardly contribute to the above integral. Thus, we can write $\phi_{12}^{(i)}\left(\vec{r}_{1}, \vec{r}_{2}\right)=\Omega^{-1 / 2} e^{\left.i \vec{p} \cdot\left[\vec{r}_{1}+\vec{r}_{2}\right) / 2\right]} \chi_{12}^{(i)}(r) Y_{00} / r$, where $r=\left|\vec{r}_{1}-\vec{r}_{2}\right|$ and $Y_{l m}$ is the $l=m=0$ spherical harmonics. The factor $\Omega^{-1 / 2} e^{i \vec{p} \cdot\left[\left(\vec{r}_{1}+\vec{r}_{2}\right) / 2\right]}$ describes the center-of-mass motion of the pair state. Then the right-hand side of Eq. (6) can be written as

$$
\sum_{i} n_{i}(\xi, \tau) \int d r s(r)\left|\chi_{12}^{(i)}(r)\right|^{2}
$$

By the above argument, $\chi_{12}(r)$ will have the form of the two-body radial wave function at short distance. In particular, in the region where $a_{s}, k_{F}^{-1} \gg r \gtrsim r_{0}$, we can write $\chi_{12}^{(i)}(r)$ $=C^{(i)}(\xi, \tau) \bar{\chi}_{12}(r)$, where

$$
\bar{\chi}_{12}(r) \equiv 1-\frac{r}{a_{s}}
$$

has been normalized in such a way that it approaches 1 in the region $a_{s}, k_{F}^{-1} \gg r \gtrsim r_{0}$. The $C^{(i)}$ 's are some constants that in principle depend on the many-body physics. Equation (6) can then be written as

$$
\begin{aligned}
& \int d \vec{r}_{1} d \vec{r}_{2} s\left(\vec{r}_{1}-\vec{r}_{2}\right)\left\langle\psi_{1}^{\dagger}\left(\vec{r}_{1}\right) \psi_{2}^{\dagger}\left(\vec{r}_{2}\right) \psi_{2}\left(\vec{r}_{2}\right) \psi_{1}\left(\vec{r}_{1}\right)\right\rangle \\
& =\sum n^{(i)}(\xi, \tau)\left|C^{(i)}(\xi, \tau)\right|^{2} \int d r s(r)\left|\bar{\chi}_{12}(r)\right|^{2} \\
& \equiv h(\xi, \tau) k_{F} N \int d r s(r)\left|\bar{\chi}_{12}(r)\right|^{2},
\end{aligned}
$$

where we have defined a positive-definite universal function

$$
h(\xi, \tau) \equiv \sum_{i} \frac{n^{(i)}(\xi, \tau)}{N k_{F}}\left|C^{(i)}(\xi, \tau)\right|^{2}>0 .
$$

The factor $k_{F}$ is inserted in order to make $h(\xi, \tau)$ a dimensionless function. The integral in Eq. (9) is a constant depending on the function $s(r)$, but it is purely a two-body quantity and can be calculated without making reference to the many-body system. In particular, it does not depend on the temperature $T$. We note further that the integral displays no singular dependence on $a_{s}$ as we approach the resonance. Thus for the discussion of many-body physics, it can be regarded as a known parameter. The intricate many-body correlations are then incorporated in one universal function $h(\xi, \tau)$ and are themselves universal. As we shall show later, at unitarity, $h(\xi=0, \tau)$ must be finite and thus we conclude Eq. (9) scales with $k_{F}$ at unitarity. We also note that a similar quantity, called contact intensity, is defined by Tan [32]. Later, in Ref. [33], Braaten and Platter have rederived some results regarding "contact intensity" using field theoretical method; see Sec. III B.

Before ending the discussion of this section, let us remind ourselves of the assumptions made so far:

(a) Only $s$-wave scattering is important. The neglect of higher angular momentum $(\hbar l)$ partial waves is justified because they are of relative order $\left(k_{F} r_{0}\right)^{2 l}$ and thus negligible as compared with $s$-wave scattering. In fact, in the model Hamiltonian considered in the literature, only $s$-wave scattering is included. 
(b) The short-range form of the pair function $\chi\left(\vec{r}_{1}-\vec{r}_{2}\right)$ is determined by two-body physics and, moreover, corresponds to only one particular form of the two-body wave function in the range $\sim r_{0}$. The former assumption is justified because of (i) the diluteness of the system $k_{F} r_{0} \ll 1$ and (ii) the "exchange hole:" the Pauli principle forbids two particles with like spin to be close to each other. The later assumption comes from energetic considerations: as long as we are interested in the many-body physics, which has a typical energy scale $\epsilon_{F}$, the relevant two-body state is the one that is closest to the zero-energy scattering state, with all the other two-body states too far away to be of any practical importance.

In the following, we shall consider a uniform system with density $n$ at temperature $T$. The interactions between particles can be written as

$$
\frac{1}{2} \sum_{i, j}\left[f\left(\vec{r}_{i}-\vec{r}_{j}\right)+g\left(\vec{r}_{i}-\vec{r}_{j}\right) \vec{S}_{i} \cdot \vec{S}_{j}\right] .
$$

Here $f(\vec{r})$ and $g(\vec{r})$ are the direct and exchange interaction, respectively. $\vec{S}$ is the spin operator of the valence electron of the atom under consideration. Experimentally, one normally works with an equal population of atoms (say ${ }^{6} \mathrm{Li}$ ) in the lowest two hyperfine states $|1\rangle$ and $|2\rangle$. To the extent that one can neglect the closed-channel component, as is the case for a broad resonance, one may replace the full interaction by an effective short-range interaction in the open channel $V_{\lambda}(\vec{r})$ $\equiv V(\vec{r}, \lambda)$, where $\lambda$ is a controlling parameter by which one can tune the scattering length $a_{s}$ [7]. However, in discussing the closed-channel population, it is necessary to introduce explicitly the inter-channel coupling term $W(\vec{r})$, which converts open-channel pair states to closed-channel molecules; see the discussion in Sec. III D.

\section{PHYSICAL QUANTITIES}

In the following, we shall discuss several physical quantities that can be expressed in terms of the universal function $h(\xi, \tau)$ and thus display universal dependence on the temperature $T$.

\section{A. Interaction energy}

The simplest physical quantity that can be cast in the form of Eq. (9) is the interaction energy of the system. According to the discussion above, since the interactions between particles are of short-range form, we can write the interaction energy per particle $\frac{\langle V\rangle}{N}$ as

$$
\begin{aligned}
& \frac{1}{N} \int d \vec{r}_{1} d \vec{r}_{2} V\left(\vec{r}_{1}-\vec{r}_{2}\right)\left\langle\psi_{1}^{\dagger}\left(\vec{r}_{1}\right) \psi_{2}^{\dagger}\left(\vec{r}_{2}\right) \psi_{2}\left(\vec{r}_{2}\right) \psi_{1}\left(\vec{r}_{1}\right)\right\rangle \\
& \quad=C_{V}\left(a_{s}\right) k_{F} h(\xi, \tau),
\end{aligned}
$$

where $C_{V}\left(a_{s}\right) \equiv \int d r V(r)\left|\bar{\chi}_{12}(r)\right|^{2}$ is a well-defined, purely two-body quantity. All the many-body dependence of interaction energy is encapsulated in the universal function $h(\xi, \tau)$. Since the interaction energy must be well-defined at unitarity, $h(\xi, \tau)$ must be finite, and moreover free of any divergence as $\xi$ approaches zero. Thus the average interaction energy per particle $\frac{\langle V\rangle}{N}$ scales as $k_{F}$ at unitarity. The interaction energy of the system depends on microscopic details of the system, even at unitarity, as is clear from the factor $C_{V}\left(a_{s}\right)$. This result should be compared with the total energy of the system, to be discussed in the next subsection, which is proportional to the Fermi energy $\epsilon_{F}$ at unitarity, independent of microscopic details.

\section{B. Universal thermodynamics}

We first consider the case at temperature $T=0$. To derive an expression for the total energy of the system, we first recall that, if $\lambda$ is the tuning parameter of the potential by which the scattering length can be varied (see the Appendix), then the relation between $a_{s}$ and $\lambda$ is given by

$$
\delta a_{s}^{-1}=-\frac{m}{\hbar^{2}}\left(\int_{0}^{\infty} d r \frac{\partial V(r, \lambda)}{\partial \lambda}\left|\bar{\chi}_{12}(r)\right|^{2}\right) \delta \lambda .
$$

On the other hand, according to the Hellmann-Feynman theorem, we have

$$
\frac{\partial E}{\partial \lambda}=\left\langle\frac{\partial V(\vec{r}, \lambda)}{\partial \lambda}\right\rangle,
$$

with the average taken over the many-body state as in Eq (6). Since $\frac{\partial V}{\partial \lambda}$ is a short-ranged function, we can use Eq. (13) to rewrite Eq. (14) in terms of $a_{s}^{-1}$; we find $[33,34]$

$$
\frac{\partial E}{\partial a_{s}^{-1}}=-\frac{\hbar^{2}}{m} N k_{F} h(\xi) .
$$

Here we have used the definition of $h(\xi)$ in Eq. (9) and $h(\xi) \equiv h(\xi, \tau)$. Or in terms of $\xi=-\left(k_{F} a_{s}\right)^{-1}$,

$$
\frac{\partial E}{\partial \xi}=\frac{\hbar^{2} k_{F}^{2}}{m} N h(\xi)=2 \epsilon_{F} N h(\xi) .
$$

Since by definition $h(\xi)$ is a positive-definite function, we find the somewhat trivial result that the ground-state energy is a monotonically increasing function of $\xi$. The boundary condition on the above differential equation is easily obtained. Consider the case in which $\xi=+\infty$. We then have a free Fermi gas with the average single-particle energy $\epsilon(\xi$ $=\infty) \equiv \frac{E}{N}=\frac{3}{5} \epsilon_{F}$. Integrating Eq. (16), we find that the singleparticle energy at zero temperature along the BEC-BCS crossover is given by

$$
\epsilon(\xi)=\frac{3}{5} \epsilon_{F}-2 \epsilon_{F} \int_{\xi}^{\infty} h\left(\xi^{\prime}\right) d \xi^{\prime},
$$

where $\epsilon(\xi) \equiv \epsilon(\xi, \tau=0)$. Intuitively, $h(\xi)$ accounts for the reduction of the single-particle energy due to interaction effects. At unitarity, $\epsilon_{0} \equiv \epsilon(\xi=0, \tau=0)=(1+\beta) \frac{3}{5} \epsilon_{F}$, so we find

$$
\beta=-\frac{10}{3} \int_{0}^{\infty} h\left(\xi^{\prime}\right) d \xi^{\prime} .
$$

Around unitarity where $\xi \ll 1$, we can obtain an expansion of the average single-particle energy in terms of $\xi$. To this end, 
we can integrate Eq. (16) from $\xi^{\prime}=0$ to $\xi^{\prime}=\xi$ and we find

$$
\epsilon(\xi)-\epsilon(\xi=0)=2 \epsilon_{F} \int_{0}^{\xi} h\left(\xi^{\prime}\right) d \xi^{\prime} .
$$

For $\xi$ close to zero, the question reduces to the expansion of $h(\xi)$. From the discussion in the previous subsection, we know $h(\xi=0)$ is finite, so we conclude the energy correction away from unitarity is linear in $\xi$ and given by

$$
\epsilon(\xi)-\epsilon(\xi=0)=2 \epsilon_{F} h(\xi=0) \xi+\cdots .
$$

At zero temperature, the value of $h(\xi=0, \tau=0)$ can be calculated using the $\epsilon$ expansion, where one has to sum over all the higher-order logarithms in order to recover the correct linear $\xi$ dependence of the energy [27]. Instead of referring back to the conditions imposed by the interaction energy on the function $h(\xi, \tau)$, one can have a direct derivation of the linear $\xi$ dependence of the energy away from unitarity by a straightforward generalization of the argument in the twobody case. This is presented in the Appendix.

The generalization of the above considerations to finite temperature is straightforward. However, it is important to bear in mind that at finite temperature, in order for Eq. (16) to be correct, we have to consider the adiabatic process, in which the entropy of the system is kept constant as we change $\xi$, i.e.,

$$
\left.\frac{\partial E}{\partial \xi}\right|_{S}=2 \epsilon_{F} N h(\xi, \tau) .
$$

At finite temperature, Eqs. (17)-(20) are still right with the replacement of $h(\xi)$ by $h(\xi, \tau)$, provided that we are considering an adiabatic process. We can use thermodynamic relations to write Eq. (16) in a more useful way in terms of free energy per particle $f(\xi, \tau)$,

$$
\left.\frac{\partial f}{\partial \xi}\right|_{\tau}=\frac{1}{N}\left\langle\frac{\partial H}{\partial \xi}\right\rangle=2 \epsilon_{F} h(\xi, \tau) .
$$

Here the temperature $\tau$ is kept constant. For $\xi \rightarrow-\infty$, we know that the system is equivalent to noninteracting Bose gas with mass $2 m$ and density $n / 2$. We denote its free energy as $F_{B}(\tau)$ and introduce $f_{B}(\tau)=F_{B}(\tau) / N$, which is half of the free energy per molecule. Correspondingly, we introduce the single-particle entropy $s_{B}(\tau)=-\frac{1}{N} \frac{\partial F_{B}}{\partial T}$ and the single-particle specific heat $c_{B}(\tau)=T \frac{\partial S_{B}}{\partial T}$. Then we can integrate Eq. (22) from $-\infty$ to $\xi$ and find

$$
f(\xi, \tau)=f_{B}(\tau)+2 \epsilon_{F} \int_{-\infty}^{\xi} h\left(\xi^{\prime}, \tau\right) d \xi^{\prime} .
$$

The entropy per particle $s$ can be written as

$$
s(\tau)=s_{B}(\tau)-2 \int_{-\infty}^{\xi} \frac{\partial h\left(\xi^{\prime}, \tau\right)}{\partial \tau} d \xi^{\prime} .
$$

Notice that at $T=0$, if we assume that the ground state is not macroscopically degenerate, then $s \equiv 0$, which suggests that

$$
\lim _{\tau \rightarrow 0} \frac{\partial h(\xi, \tau)}{\partial \tau}=0, \quad \forall \xi .
$$

This means that apart from a constant term (a function of $\xi$ ), the expansion of $h(\xi, \tau)$ must have the following form:

$$
h(\xi, \tau)=h(\xi)+A(\xi) \tau^{n}+\cdots, \quad n>1,
$$

where $A(\xi)$ is some well-behaved function of $\xi$. The singleparticle specific heat can be directly obtained by using $c$ $=1 / N T \frac{\partial S}{\partial T}=\tau \frac{\partial s}{\partial \tau}$,

$$
c(\tau)=c_{B}(\tau)-\tau \int_{-\infty}^{\xi} \frac{\partial^{2} h\left(\xi^{\prime}, \tau\right)}{\partial \tau^{2}} d \xi^{\prime} .
$$

Before we conclude this subsection, we would like to derive a simple relation between the chemical potential $\mu$ and the average single-particle energy $\epsilon$ at zero temperature and thus write down the zero-temperature chemical potential in terms of $h(\xi)$. Note that at $T=\tau=0$, we can write the singleparticle energy as $\epsilon=\epsilon_{F} f_{E}(\xi)$. Thus using the thermodynamic relation $P=-\frac{\partial E}{\partial V}$ and $E=N \epsilon$, we find

$$
p=n^{2} \frac{\partial \epsilon}{\partial n}=n^{2} \frac{\partial\left[\epsilon_{F} f(\xi)\right]}{\partial n} .
$$

Using the relation $n=\frac{k_{F}^{3}}{3 \pi^{2}}$, we can make a change of variable to $k_{F}$ and write Eq. (28) as $p=\frac{1}{3} n k_{F} \frac{\partial \epsilon}{\partial k_{F}}$. Now, using the expression $\epsilon=\epsilon_{F} f(\xi)$ and the fact that $f(\xi)$ only depends on the combination $\xi \equiv-\left(k_{F} a_{S}\right)^{-1}$, we obtain, $p=\frac{2}{3} n \epsilon+\frac{1}{3} n a_{s} \frac{\partial \epsilon}{\partial a_{s}}$ [35]. We consider the situation when the density of the system is fixed, and we write the above expression as $\epsilon=\frac{3}{2} \frac{p}{n}+\frac{1}{2} \xi \frac{\partial \epsilon}{\partial \dot{\xi}}$. At $T=0$, we have the thermodynamic relation $\epsilon=-\frac{p}{n}+\mu$, where $p$ is the pressure and $n$ is the average density. We find

$$
\frac{5}{2} \epsilon=\frac{3}{2} \mu+\frac{1}{2} \xi \frac{\partial \epsilon}{\partial \xi} .
$$

The above expression is very general and works along the whole BEC-BCS crossover provided that the density $n$ is kept constant. In the extreme BEC limit, the single-particle energy equals the chemical potential: $\epsilon=\mu=-\frac{\hbar^{2}}{2 m a_{s}^{2}}$. One verifies that this is satisfied by Eq. (29). In the BCS limit, the first-order correction to the energy will be of order $a_{s}$, namely of order $\xi^{-1}$, coming from Hartree-Fock corrections. Since $\frac{\partial \epsilon}{\partial \dot{\xi}}=-\xi^{-1} \frac{\partial \epsilon}{\partial \xi^{-1}}=-a_{s} \frac{\partial \epsilon}{\partial a_{s}} \rightarrow 0$ when $a_{s} \rightarrow 0$, we thus recover the usual relation between chemical potential $\mu$ and the average single-particle energy of the free Fermi gas $\epsilon$ $=\frac{3}{5} \mu$. At unitarity, $\xi=0$, we find again the free Fermi gas result $\epsilon=\frac{3}{5} \mu$, if we assume that the energy is continuous at unitarity.

Finally, using Eq. (16), we can write the zero-temperature chemical potential in terms of $h(\xi)$ as 


$$
\mu(\xi)=\epsilon_{F}\left(1-\frac{2}{3} \xi h(\xi)-\frac{10}{3} \int_{\xi}^{\infty} h\left(\xi^{\prime}\right) d \xi^{\prime}\right) .
$$

Setting $\xi=0$, we recover Eq. (18) since at unitarity $\mu=(1$ $+\beta) \epsilon_{F}$.

\section{C. rf-spectroscopy shift $\overline{\delta \omega}$}

One of the early experiments that indicated the appearance of a new low-temperature quantum state in ultracold Fermi gases was the radio-frequency spectroscopic experiment carried out by the Innsbruck group [36]. The experiment works with the lowest two hyperfine-Zeeman states (|1 and $|2\rangle)$ of ${ }^{6} \mathrm{Li}$. A radio-frequency field is applied to drive atoms from state $|2\rangle$ to $|3\rangle$. It is found that at high temperature, the frequency of the rf field coincides with the bare atomic transition from $|2\rangle$ to $|3\rangle$, while at low temperature, there is an up-shift in the rf frequency, which indicates that the system is in a new quantum state. The interpretation of the experiment is fairly complicated. On the one hand, the experiment was not carried out in the linear-response regime, so, strictly speaking, a simple-minded sum rule calculation is not applicable; on the other hand, the quasiparticle tunneling picture seems to obscure the fundamental consideration regarding the $\mathrm{SU}(2)$ (non)invariance of the interparticle interaction, which is of crucial importance in explaining the early NMR data of ${ }^{3} \mathrm{He}$ [37]. These two pictures are discussed in detail in a recent paper by Leskinen et al., to which we refer for details [38]. It is also understood that the full understanding of $\mathrm{rf}$ spectroscopy requires a proper treatment of the final-state interactions [39-47]. Here we follow Ref. [46], where a unified treatment is given for the rf spectroscopy along the BEC-BCS crossover within the linear-response theory. It has been shown there that the average shift of the rf spectroscopy is given by the following expression $[43,44,46]$, in the present notation:

$$
\begin{aligned}
\overline{\delta \omega} & =\frac{G(H)+J(H)}{\hbar N_{2}} \int g\left(\vec{r}_{1}-\vec{r}_{2}\right)\left\langle\psi_{1}^{\dagger}\left(\vec{r}_{1}\right) \psi_{2}^{\dagger}\left(\vec{r}_{2}\right) \psi_{2}\left(\vec{r}_{2}\right) \psi_{1}\left(\vec{r}_{1}\right)\right\rangle \\
& =\frac{G(H)+J(H)}{\hbar} C_{g}\left(a_{s}\right) 2 k_{F} h(\xi, \tau),
\end{aligned}
$$

where $C_{g}\left(a_{s}\right)=\int g(r)\left|\bar{\chi}_{12}(r)\right|^{2} d r$ and the functions $G(H)$ and $J(H)$ are given in Ref. [46], $g(\vec{r})$ is the exchange interaction, and $N_{2}=N / 2$ is the particle number in the hyperfine-Zeeman state $|2\rangle$. Again $C_{g}\left(a_{s}\right)$ is independent of temperature $T$ and we conclude that the average rf shift has the same temperature dependence as the interaction energy at arbitrary $\xi$. Note that $\overline{\delta \omega}$ scales with $k_{F}$ at unitarity.

\section{Closed-channel fraction}

One of the key physical quantities in the BEC-BCS crossover using Feshbach resonance is the population in the closed channel. This quantity has been experimentally determined using an optical molecular spectroscopic technique [19]. The experiment uses ${ }^{6} \mathrm{Li}$ atoms in their lowest two hyperfine states $|1\rangle$ and $|2\rangle$, in which they interact primarily through the electronic triplet potential. On the other hand, the associated closed-channel molecules induced by the Feshbach resonance interact primarily through the much deeper electronic singlet potential. In the experiment, a laser beam induces an electric dipole transition between the closedchannel molecular state $\left(X^{1} \Sigma_{g}^{+}, \nu=38\right)$ to another closedchannel molecular state with $\nu=68, A^{1} \Sigma_{u}^{+}$. At low temperature, it is inferred from the loss signal that on the BCS side of the resonance, there is a finite fraction of closed-channel molecules that is not supported by the two-body physics. Thus it is suggested that the many-body quantum state must have nontrivial two-particle correlations like those in the BCS state to account for the observed one-body decay in the BCS side [19]. To address the closed-channel fraction theoretically [48-50], let us first identify the inter-channel coupling $W(\vec{r})$ from the bare interactions between the two atoms, $U(\vec{r})=f(\vec{r})+g\left(\vec{r}_{1}\right) \vec{S}_{1} \cdot \vec{S}_{2} . f(\vec{r})$ and $g(\vec{r})$ are the direct and exchange interaction, respectively. We restrict ourselves to the case in which there are only two channels involved, namely an open channel with atoms in the lowest two hyperfineZeeman states $|1\rangle$ and $|2\rangle$ and the corresponding closed channel with atoms in hyperfine-Zeeman states $|1\rangle$ and $|4\rangle$. Notice that one of the hyperfine-Zeeman states is common to the open and closed channels. We shall denote the interaction potential in the open and closed channel by $V_{o}(\vec{r})$ $=\langle 12|U| 12\rangle$ and $V_{c}(\vec{r})=\langle 14|U| 14\rangle$, respectively. Now, the inter-channel coupling can be written as

$$
W\left(\vec{r}_{1}-\vec{r}_{2}\right)=g\left(\vec{r}_{1}-\vec{r}_{2}\right)\left\langle 14\left|\vec{S}_{1} \cdot \vec{S}_{2}\right| 12\right\rangle,
$$

where $|\alpha \beta\rangle$ denotes a spin-singlet state $|\alpha \beta\rangle=\left(|\alpha\rangle_{1}|\beta\rangle_{2}\right.$ $\left.-|\beta\rangle_{1}|\alpha\rangle_{2}\right) / \sqrt{2}$. The Hamiltonian is given by

$$
\begin{aligned}
\mathcal{H}= & \sum_{\alpha} \int d \vec{r} \psi_{\alpha}^{\dagger}(\vec{r})\left(-\frac{\hbar^{2}}{2 m} \nabla^{2}-\mu_{\alpha}+E_{\alpha}\right) \psi_{\alpha}(\vec{r}) \\
& +\frac{1}{2} \sum_{\alpha \beta \gamma \delta} \int d \vec{r}_{1} d \vec{r}_{2} \psi_{\alpha}^{\dagger}\left(\vec{r}_{1}\right) \psi_{\beta}^{\dagger}\left(\vec{r}_{2}\right) \\
& \times U_{\alpha \beta \gamma \delta}\left(\vec{r}_{1}-\vec{r}_{2}\right) \psi_{\gamma}\left(\vec{r}_{2}\right) \psi_{\delta}\left(\vec{r}_{1}\right),
\end{aligned}
$$

where $\mu_{\alpha}$ is the chemical potential of the $\alpha$ component and $U_{\alpha \beta \gamma \delta}(\vec{r})=f(\vec{r}) \delta_{\alpha \delta} \delta_{\beta \gamma}+g(\vec{r})\left\langle\alpha\left|\vec{S}_{1}\right| \delta\right\rangle \cdot\left\langle\beta\left|\vec{S}_{2}\right| \gamma\right\rangle . \quad E_{\alpha}$ is the energy of the hyperfine-Zeeman state $|\alpha\rangle$. Note that to the extent that the particle number in any one hyperfine-Zeeman level is conserved in the absence of the laser beam, that is, if we neglect any decay of atoms from one hyperfine-Zeeman state to another, we have only two independent chemical potentials, $\mu_{1}$ and $\mu_{2}=\mu_{4}$, corresponding to the two separately conserved quantities $N_{1}=N / 2$ and $N_{2}+N_{4}=N / 2$. To address the population of the closed channel in a many-body system, we look at the equation of motion for a product of two Fermi operators, $\psi_{\alpha}\left(\vec{r}_{1}, t\right) \psi_{\beta}\left(\vec{r}_{2}, t\right)$, 


$$
\begin{aligned}
i \hbar \frac{\partial\left(\psi_{\alpha}\left(\vec{r}_{1}, t\right) \psi_{\beta}\left(\vec{r}_{2}, t\right)\right)}{\partial t}= & \left(-\frac{\nabla_{1}^{2}}{2 m}-\mu_{\alpha}+E_{\alpha}-\frac{\nabla_{2}^{2}}{2 m}-\mu_{\beta}+E_{\beta}\right) \psi_{\alpha}\left(\vec{r}_{1}\right) \psi_{\beta}\left(\vec{r}_{2}\right)+\sum_{\gamma \delta} U_{\alpha \beta \delta \gamma}\left(\vec{r}_{1}-\vec{r}_{2}\right) \psi_{\gamma}\left(\vec{r}_{1}\right) \psi_{\delta}\left(\vec{r}_{2}\right) \\
& +\sum_{\beta^{\prime} \gamma \delta} \int d^{3} \vec{r}^{\prime} U_{\alpha \beta^{\prime} \gamma \delta}\left(\vec{r}_{1}-\vec{r}^{\prime}\right) \psi_{\beta^{\prime}}^{\dagger}\left(\vec{r}^{\prime}\right) \psi_{\gamma}\left(\vec{r}^{\prime}\right) \psi_{\delta}\left(\vec{r}_{1}\right) \psi_{\beta}\left(\vec{r}_{2}\right) \\
& +\sum_{\beta^{\prime} \gamma \delta} \int d^{3} \vec{r}^{\prime} U_{\beta \beta^{\prime} \gamma \delta}\left(\vec{r}_{2}-\vec{r}^{\prime}\right) \psi_{\beta^{\prime}}^{\dagger}\left(\vec{r}^{\prime}\right) \psi_{\gamma}\left(\vec{r}^{\prime}\right) \psi_{\alpha}\left(\vec{r}_{1}\right) \psi_{\delta}\left(\vec{r}_{2}\right) .
\end{aligned}
$$

Note that since both $f(\vec{r})$ and $g(\vec{r})$ are short-range functions of order $r_{0}$, it is clear from Eq. (34) that the conversion from an open-channel pair state to a closed-channel molecular state occurs only at short distance, i.e., $\left|\vec{r}_{1}-\vec{r}_{2}\right| \sim r_{0}$. It follows then that the last two terms in Eq. (34) are of minor importance as compared with the other terms since they involve another coordinate $\vec{r}^{\prime}$ that should be close to $\vec{r}_{1}$ or $\vec{r}_{2}$ and thus bring up extra factors of $k_{F} r_{0}$. They provide either an effective background potential or introduce pair states other than the ones under consideration $(|12\rangle$ and $|14\rangle)$, which are relatively unimportant and thus not of concern here. In the following, we shall neglect the last two terms in Eq. (34). Now, taking Eq. (34) to act on the ground state or thermal ensemble, we find the coupled equation of motion of a state with two holes in it. Let us denote this state by $\phi_{o}\left(\vec{r}_{1}, \vec{r}_{2}\right)$ and $\phi_{c}\left(\vec{r}_{1}, \vec{r}_{2}\right)$, where subscript $o$ refers to $\alpha=1$, $\beta=2$ of the open channel and $c$ refers to $\alpha=1, \beta=4$ of the closed channel. We assume that the rotational degrees of freedom of the closed-channel molecules are not excited at low temperature and remain in a relative $s$-wave state. In that case, since $W(\vec{r})$ is in fact isotropic in space, only the $s$-wave components of $\phi_{0}\left(\vec{r}_{1}, \vec{r}_{2}\right)$ are important in discussing the population of the closed-channel molecules. On the other hand, only $s$-wave pair states in the open channel can be converted by a short-range potential $W(\vec{r})$ to closed-channel molecules, as is clear from the structure of Eq. (35). By performing a Fourier transform with respect to the center-ofmass coordinate $2 \vec{R}=\vec{r}_{1}+\vec{r}_{2}$ and time, we find the following coupled equation:

$$
\begin{aligned}
& \left(\omega+\frac{\nabla^{2}}{m}+\mu_{1}+\mu_{2}-E_{K}-V_{o}(\vec{r})\right) \phi_{o}(\vec{r} ; \vec{K}, \omega) \\
& =W(\vec{r}) \phi_{c}(\vec{r} ; \vec{K}, \omega), \\
& \left(\omega+\frac{\nabla^{2}}{m}+\mu_{1}+\mu_{4}-E_{K}-\widetilde{\delta}_{c}-\epsilon_{0}-V_{c}(\vec{r})\right) \phi_{c}(\vec{r} ; \vec{K}, \omega) \\
& =W(\vec{r}) \phi_{o}(\vec{r} ; \vec{K}, \omega) .
\end{aligned}
$$

Here $-\epsilon_{0}$ is the energy of the molecular state in the closed channel relative to its asymptotic energy $E_{2}+E_{4}$ when the two atoms are far away from each other, $E_{\vec{K}}=\hbar^{2} \vec{K}^{2} / 4 m$ is the center-of-mass kinetic energy of a pair of atoms, and $\widetilde{\delta}_{c}$ $=E_{4}-E_{2}-\epsilon_{0}$ is the so-called bare detuning from the Feshbach resonance. In the case of a broad Feshbach resonance, it is much larger than the many-body energy scale, in particular, $\tilde{\delta}_{c} \gg \epsilon_{F}$. Even though the form of the coupled equation (35) is the same as that for the two-body case, the manybody physics does play an important role as it determines the normalizations for the function $\phi_{o}\left(\vec{r}_{1}, \vec{r}_{2}\right)$ and $\phi_{c}\left(\vec{r}_{1}, \vec{r}_{2}\right)$. Let us note one feature of Eq. (35) that is conceptually important: Since the inter-channel coupling depends only on the relative coordinate, the center-of-mass momentum $\vec{K}$ is a good quantum number and we conclude that the pair distributions in the open and closed channel are connected by Eq. (35) due to the superposition nature of the open-channel pairs and closed-channel molecules. It is thus in general not permissible to assign independent momentum distributions to the closed-channel molecules and open-channel pairs; specifying either one of them suffices to fix the other through Eq. (35). Also note that if we neglect, as we shall do later, the relatively unimportant factor $E_{K}$ as compared with $\widetilde{\delta}_{c}$, the coupled equation (35) is identical for different $\vec{K}$ states. That implies that, whatever the center-of-mass momentum $\vec{K}$ is for the open-channel pair state, the inter-channel coupling always induces the same amount of closed-channel molecules associated with it. The irrelevance of finite $\vec{K}$ states in discussing closed-channel molecule formation can again be understood as a result of its high-energy character, that is, the process occurs only at short distance, of order $r_{0}$, and therefore many-body physics is quite incapable of modifying it.

Let us then introduce the Green function for the closedchannel equation,

$$
\left(\omega+\frac{1}{m} \frac{d^{2}}{d r^{2}}-V_{c}(r)\right) G\left(r, r^{\prime}\right)=\delta\left(r-r^{\prime}\right),
$$

where $G\left(r, r^{\prime}\right)$ is given by

$$
G\left(r, r^{\prime}\right)=\sum_{n} \frac{\chi_{n}^{*}(r) \chi_{n}\left(r^{\prime}\right)}{\omega-E_{n}} \approx \frac{\chi_{0}^{*}(r) \chi_{0}\left(r^{\prime}\right)}{\omega+\epsilon_{0}}
$$

where $\chi_{0}(r)$ is the normalized eigenfunction in the closed channel with energy $-\epsilon_{0}$. Using Eq. (37) to integrate the closed-channel equation, we find 


$$
\begin{aligned}
\phi_{c}(r ; \vec{K}, \omega)= & \frac{1}{\omega+\mu_{1}+\mu_{4}-E_{K}-\widetilde{\delta}_{c}} \\
& \times \int d r^{\prime} \chi_{0}^{*}(r) \chi_{0}\left(r^{\prime}\right) W\left(r^{\prime}\right) \phi_{o}\left(r^{\prime} ; \vec{K}, \omega\right) .
\end{aligned}
$$

Again, we see that since $W\left(r^{\prime}\right)$ is short-ranged, the integration only picks up the short-range part of the pair wave function. An important question is the appropriate value for $\omega$. It is clear that the state obtained by removing two particles in states $|1\rangle$ and $|2\rangle$ does not in general correspond to the eigenstate (or thermal equilibrium) for the $N-2$ particle system. It is, however, clear that $\omega$ will be centered around $-\left(\mu_{1}+\mu_{2}\right)$, corresponding to the energy difference between the ground states for the $(N-2)$ - and $N$-particle state. The spread of $\omega$ will be in general smaller than the Fermi energy even at resonance. In the case of a wide resonance, it is known that $\tilde{\delta}_{c}$ is much larger than the many-body scale, so if we approximate the denominator in the above equation by $\widetilde{\delta}_{c}$ and make a Fourier transform with respect to $\vec{K}$, we find

$$
\phi_{c}(r, \vec{R})=-\frac{1}{\widetilde{\delta}_{c}} \int d r^{\prime} \chi_{0}^{*}(r) \chi_{0}\left(r^{\prime}\right) W\left(r^{\prime}\right) \phi_{o}\left(r^{\prime}, \vec{R}\right) .
$$

This implies that the number of molecules in the closed channel $N_{C}$ is given by

$$
\begin{aligned}
N_{C} & =\int d r d \vec{R} \phi_{c}(r, \vec{R})^{*} \phi_{c}(r, \vec{R}) \\
& =\left(\frac{1}{\widetilde{\delta}_{c}}\right)^{2} \int d R d r^{\prime} d r^{\prime \prime} K\left(r^{\prime}, r^{\prime \prime}\right) \phi_{o}\left(r^{\prime}, \vec{R}\right) \phi_{o}^{*}\left(r^{\prime \prime}, \vec{R}\right),
\end{aligned}
$$

where $K\left(r^{\prime}, r^{\prime \prime}\right)=\chi_{0}\left(r^{\prime}\right) \chi_{0}^{*}\left(r^{\prime \prime}\right) W\left(r^{\prime}\right) W\left(r^{\prime \prime}\right)$. It is clear that the kernel $K\left(r^{\prime}, r^{\prime \prime}\right)$ is a short-range function in both $r^{\prime}$ and $r^{\prime \prime}$, and that $\phi_{o}\left(r^{\prime}, \vec{R}\right) \phi_{o}^{*}\left(r^{\prime \prime}, \vec{R}\right)$ corresponds to the $s$-wave part of the following density matrix: $\left\langle\psi_{1}^{\dagger}\left(\vec{R}+\frac{\vec{r}^{\prime}}{2}\right) \psi_{2}^{\dagger}\left(\vec{R}-\frac{\vec{r}^{\prime}}{2}\right) \psi_{2}(\vec{R}\right.$ $\left.\left.-\frac{\vec{r}^{\prime \prime}}{2}\right) \psi_{1}\left(\vec{R}+\frac{\vec{r}^{\prime \prime}}{2}\right)\right\rangle$. Using the same decomposition as before, we find

$$
f_{c} \equiv \frac{N_{C}}{N}=\left(\frac{1}{\widetilde{\delta}_{c}}\right)^{2} k_{F} C_{K}\left(a_{s}\right) h(\xi, \tau),
$$

where we have used Eq. (9) and defined $C_{K}\left(a_{s}\right)$ $=\int d r^{\prime} d r^{\prime \prime} \bar{\chi}\left(r^{\prime}\right)^{*} K\left(r^{\prime}, r^{\prime \prime}\right) \bar{\chi}\left(r^{\prime \prime}\right)$. Noting that the dimension of $C_{K}\left(a_{s}\right)$ is given by $[E]^{2}[L]$, we can define a length scale $l_{c}$ by

$$
l_{c} \equiv \frac{C_{K}\left(a_{s}\right)}{\widetilde{\delta}_{c}^{2}}=\frac{1}{\widetilde{\delta}_{c}^{2}} \int d r^{\prime} d r^{\prime \prime} \bar{\chi}\left(r^{\prime}\right)^{*} K\left(r^{\prime}, r^{\prime \prime}\right) \bar{\chi}\left(r^{\prime \prime}\right) .
$$

$l_{c}$ is entirely determined by the two-body physics. Note that since $W(\vec{r})$ in Eq. (32) has the factor $\left\langle 12\left|\vec{S}_{1} \cdot \vec{S}_{2}\right| 14\right\rangle$ and, as a result, the quantity $l_{c}$ is magnetic-field-dependent, its dependence can be calculated straightforwardly. We find

$$
l_{c}=L(H) \frac{1}{\widetilde{\delta}_{c}^{2}} \int d r^{\prime} d r^{\prime \prime} \bar{\chi}\left(r^{\prime}\right)^{*} \widetilde{K}\left(r^{\prime}, r^{\prime \prime}\right) \bar{\chi}\left(r^{\prime \prime}\right),
$$

where $\tilde{K}\left(r^{\prime}, r^{\prime \prime}\right)=\chi_{0}\left(r^{\prime}\right) \chi_{0}^{*}\left(r^{\prime \prime}\right) g\left(r^{\prime}\right) g\left(r^{\prime \prime}\right)$ is magnetic-fieldindependent and function $L(H)$ is given by

$$
L(H)=\frac{\left(1-2 \alpha^{2}\right)^{2} \beta^{2}}{4\left(1+\alpha^{2}\right)^{2}\left(1+\beta^{2}\right)^{2}},
$$

where the parameters $\alpha$ and $\beta$ depend on magnetic field and are given in Ref. [46]. Finally, we can rewrite the molecular fraction in the closed channel as

$$
f_{c}=k_{F} l_{c} h(\xi, \tau) \text {. }
$$

At unitarity, $f_{c}$ scales with $k_{F}$. It can be shown that Eq. (45) is equivalent to that given in Ref. [51] [their Eq. (25)] by using their definition of $R_{*}$ [their Eq. (26)].

In the following, we shall illustrate the above general considerations in the "naive" BCS-ansatz, properly generalized to include the closed-channel component. Note that one of the spin states $(|1\rangle)$ is common to the open and closed channels,

$$
|\mathrm{BCS}\rangle=\sum_{\vec{k}}\left(u_{\vec{k}}+v_{\vec{k}} a_{\vec{k} 1}^{\dagger} a_{-\vec{k} 2}^{\dagger}+w_{\vec{k}} a_{\vec{k} 1}^{\dagger} a_{-\vec{k} 4}^{\dagger}\right)|\mathrm{vac}\rangle,
$$

where $u_{\vec{k}}, v_{\vec{k}}$, and $w_{\vec{k}}$ are the usual variational parameters, satisfying $\left|u_{\vec{k}}\right|^{2}+\left|v_{\vec{k}}\right|^{2}+\left|w_{\vec{k}}\right|^{2}=1 . \quad a_{\vec{k} i}^{\dagger}$ is the creation operator for a particle in the hyperfine-Zeeman state $|i\rangle$ with momentum $\vec{k}$. The corresponding pair wave functions that are relevant in Eq. (35) are $F_{\vec{k}}^{o}=u_{\vec{k}} v_{\vec{k}}$ and $F_{\vec{k}}^{c}=u_{\vec{k}} w_{\vec{k}}$. Let us concentrate only on the $\vec{K}=0$ pair state in the system since it corresponds to macroscopic occupation in the BCS state [cf. also the discussion after Eq. (35)]. Denoting the Fourier transform of $F_{\vec{k}}^{o}$ and $F_{\vec{k}}^{c}$ as $F^{o}(\vec{r}) \equiv \sum_{\vec{k}} u_{\vec{k}} v_{\vec{k}} e^{i \vec{k} \cdot \vec{r}}$ and $F^{c}(\vec{r})$ $\equiv \sum_{\vec{k}} u_{\vec{k}} w_{\vec{k}} e^{i \vec{k} \cdot \vec{r}}$ and setting $\omega=-\left(\mu_{1}+\mu_{2}\right)$, we find that the coupled Eqs. (35) take the form

$$
\begin{gathered}
\left(\frac{\nabla^{2}}{m}-V_{o}(\vec{r})\right) F^{o}(\vec{r})=W(\vec{r}) F^{c}(\vec{r}), \\
\left(\frac{\nabla^{2}}{m}-\widetilde{\delta}_{c}-\epsilon_{0}-V_{c}(\vec{r})\right) F^{c}(\vec{r})=W(\vec{r}) F^{o}(\vec{r}) .
\end{gathered}
$$

This coupled equation is exactly the same form as that in the two-body case (see, for example, Ref. [7]). We can follow the derivation there, or more straightforwardly we can replace $\phi_{o}(r, \vec{R})$ with $\Omega^{-1 / 2} F^{o}(r)$, where $\Omega^{-1 / 2}$ accounts for the center-of-mass motion of the pair and $F^{o}(r)$ is the radial part of the pair wave function $F^{o}(\vec{r})$. The spatial dependence of $F^{o}(\vec{r})$ is given, within the crossover model [46], by

$$
F^{o}(\vec{r})=\frac{m \Delta}{4 \pi \hbar^{2}} \frac{1-r / a_{s}}{r}
$$

We find that the density of atoms in closed channel, $n_{c}$, is given by 


$$
n_{c}=l_{c}\left(\frac{m \Delta}{\sqrt{4 \pi} \hbar^{2}}\right)^{2} .
$$

Using $n=k_{F}^{3} / 3 \pi^{2}$, we obtain the fraction of particles in the closed channel $f_{c}$,

$$
f_{c} \equiv \frac{n_{c}}{n}=\frac{3 \pi}{16} k_{F} l_{c}\left(\frac{\Delta}{\epsilon_{F}}\right)^{2} .
$$

If we compare the above equation with Eq. (45), we find $h(\xi, \tau)=3 \pi / 16\left(\Delta / \epsilon_{F}\right)^{2}$. Thus within the "naive" ansatz, the fraction of particles in the closed channel is proportional to $\Delta^{2}$ and, moreover, at resonance, scales with $k_{F}$. In the extreme BEC limit, we know $\Delta=4 \epsilon_{F} / \sqrt{3 \pi k_{F} a_{s}}$ and thus

$$
f_{c}=\frac{l_{c}}{a_{s}},
$$

independent of many-body physics, as it should be intuitively.

Before ending this section, let us mention the work in the literature on the problem of the closed-channel fraction. In the work by Javanainen et al. [50], it is assumed that the Feshbach-induced bosons in the closed channel are condensed in the $\vec{K}=0$ state. This can be regarded as a limiting case of the calculation by Chen et al. [48] in which Feshbach molecules are included in a nonzero temperature generalization of the conventional "naive" ansatz. The conclusions obtained in [48] are in agreement with our general analysis. For example, it is shown in Ref. [48] that the fraction of condensed bosons scales with $k_{F}$ at unitarity, and within their approximation, the number of closed-channel molecules (named Feshbach molecules in Ref. [48]) is proportional to $\Delta_{s c}^{2}$ [our $\Delta$ in Eq. (49) above], while the number of noncondensed molecules is proportional to $\Delta_{p g}^{2}$ [Eq. (9) in [48]]. $\Delta_{p g}$ describes noncondensed Fermion pairs, which are of course included in the general definition of the function $h(\xi, \tau)$. Thus, it is clear that the general structure of the conclusions is the same in both approaches. However, as emphasized before, as a result of the coupled nature of Eq. (35), the momentum distribution of the open-channel pair states dictates the momentum distribution of the closed-channel molecules (Feshbach molecules). While this feature is explicit at the Hamiltonian level of the two-channel model, it is in general not enforced in the actual calculations [see, for example, Eq. (94) in Ref. [20]]. To illustrate the point, let us look at the unitarity limit at $T=0$, where we know that a fraction of the Fermi pairs is not condensed; thus the induced closedchannel molecules associated with them will have nonzero momentum, far from being condensed in the $\vec{K}=0$ state.

\section{CONCLUSIONS}

By exploiting the diluteness of the ultracold Fermi gas, we have shown that, in considering various physical quantities of the system, it is possible to lump all the many-body dependence into a single universal function $h(\xi, \tau)$. A particular physical quantity may be universal, irrespective of microscopic details (e.g., the form of the interaction potential), in which case one should be able to express it entirely in terms of $h(\xi, \tau)$, as in the case of the average singleparticle energy of the system, Eq. (17). Other physical quantities are not universal and there are explicit dependences on the interaction, other than that incorporated in the function $h(\xi, \tau)$. However, those dependences can be dealt with using only the two-body physics. In this case, it is possible to show the universal temperature dependence of the physical quantities. For convenience, let us summarize these two types of behavior in the following:

(a) Universal dependence on $\xi$ and $\tau$. It is understood that universal here means that all the interaction and temperature dependences are captured in one function, $h(\xi, \tau)$. The primary example is the single-particle energy of the system, Eq. (17). Physical quantities that can be directly derived from energy will be in this category as well, for example the speed of sound $c$ and the chemical potential $\mu$ in Eq. (30).

(b) Universal temperature dependence. In this case, the physical quantities will have identical temperature dependence inherited from $h(\xi, \tau)$. Those physical quantities include the interaction energy of the system Eq. (12), the average radiofrequency spectroscopic shift, Eq. (31), and the molecular fraction in the closed channel, Eq. (45).

In actual experiment, there is always an external confining potential that renders the system inhomogeneous. The question of universality is then more delicate. However, the argument given in Sec. II is still valid provided the scale over which the confining potential varies is much larger than the range of the potential. This is well satisfied in the experiments. The universal function $h(\xi(\vec{r}), \tau)$ will depend on position $\vec{r}$ through local Fermi vector $k_{F}(\vec{r})$. The temperature dependence of the physical quantities listed in category (b) above will still have universal temperature dependence even in a trap.

Note added. Recently, we became aware of the work of Werner et al. [51], who performed a similar analysis for the closed-channel molecule fraction. Of particular interest is their definition of a universal function that is identical to our $h(\xi, \tau)$; see their Eq. (14). As compared with the paper by Werner et al., we have made an effort to connect different physical quantities together and emphasized the universal temperature dependences of the physical quantities. In addition, we have discussed in detail the physical origin of the universal function. See, however, their paper for a discussion of the tail of the momentum distribution (their Sec. III B).

\section{ACKNOWLEDGMENT}

This work was supported by the National Science Foundation under Grant No. NSF-DMR-03-50842.

\section{APPENDIX A: EXPANSION OF GROUND-STATE ENERGY AROUND UNITARITY}

In this appendix, we discuss how to establish the linear dependence of the ground-state energy $\epsilon(\xi)$ on $\xi$ around unitarity. Before we start with the many-body problem, it is instructive to look at the two-body problem for guidance. For more details, see Ref. [7]. 
Let us consider two atoms of mass $m$, interacting via a central potential $V_{\lambda}(r)$ which can be tuned by a parameter $\lambda$. The wave function for the relative motion $\chi_{\lambda}(r)$ satisfies the following time-independent Schrödinger equation [12]:

$$
-\frac{\hbar^{2}}{2 m_{r}} \frac{d^{2}}{d r^{2}} \chi_{\lambda}(r)+V_{\lambda}(r) \chi_{\lambda}(r)=E \chi_{\lambda}(r),
$$

where $m_{r}=m / 2$ is the reduced mass. Note that the normalization of $\chi_{\lambda}(r)$ is arbitrary at the moment. Let us fix this by requiring that in the region $r \gg r_{0}$,

$$
\chi_{\lambda}(r)=1-\frac{r}{a_{s}},
$$

where $r_{0}$ is the range of the two-body potential $V_{\lambda}(r)$. Consider the critical potential $V_{\lambda_{c}}$, for which $a_{s}=\infty$, and denote the corresponding radial wave function by $\chi_{0} \cdot \chi_{0}=1$ for $r$ $\gg r_{0}$. For zero-energy scattering, we have

$$
-\frac{\hbar^{2}}{2 m_{r}} \frac{d^{2}}{d r^{2}} \chi_{0}(r)+V_{\lambda_{c}}(r) \chi_{0}(r)=0 .
$$

Now, multiplying Eq. (A1) by $\chi_{0}$ (setting $E=0$ on the righthand side as well for zero-energy scattering) and multiplying Eq. (A3) by minus $\chi_{\lambda}$, we find, using Green's theorem and integrating up to $r_{0}$,

$$
\begin{aligned}
& \left.\left(\frac{d \chi_{0}(r)}{d r} \chi_{\lambda}(r)-\chi_{0}(r) \frac{d \chi_{\lambda}(r)}{d r}\right)\right|_{r_{0}} \\
& =-\frac{m}{\hbar^{2}} \int_{0}^{\infty} d r\left[V_{\lambda}(r)-V_{\lambda_{c}}(r)\right] \chi_{0}(r) \chi_{\lambda}(r) .
\end{aligned}
$$

Since both $V_{\lambda}(r)$ and $V_{\lambda_{c}}(r)$ are short-range functions, we can safely replace $\chi_{\lambda}(r)$ with $\chi_{0}(r)$ around resonance since they are identical for $r \lesssim r_{0}$. Using the explicit form of $\chi_{\lambda}(r)$ and $\chi_{0}(r)$, we find, for an infinitesimal change of $\lambda$,

$$
\delta a_{s}^{-1}=-\frac{m}{\hbar^{2}}\left(\left.\int_{0}^{\infty} d r \frac{\partial V_{\lambda}(r)}{\partial \lambda}\right|_{\lambda=\lambda_{c}}\left|\chi_{0}(r)\right|^{2}\right) \delta \lambda .
$$

Now, let us consider the many-body case. We shall be interested in a system with $N / 2$ spin-up atoms with coordinate denoted by $\vec{x}_{i}$, and $N / 2$ spin-down atoms with coordinate denoted by $\vec{y}_{i}, i=1,2, \ldots, N / 2$. The interaction between spin-up atom $i$ and spin-down atom $j$ takes the form

$$
V_{\lambda}\left(\left|\vec{x}_{i}-\vec{y}_{j}\right|\right),
$$

where $\lambda$ is a tuning parameter as in the two-body case. It determines the asymptotic behavior of the many-body wave function in the range $r_{0} \ll r \lesssim a_{s}, k_{F}^{-1}$. We denote the corresponding spatial many-body wave function as $\Psi_{\lambda}\left(\vec{x}_{1}, \vec{x}_{2}, \ldots, \vec{y}_{1}, \vec{y}_{2}, \ldots\right)$. In general, one is not allowed to write down a pure spatial wave function with spin part totally decoupled from it. However, since we are only interested in the energetics of the system, for which the spin index is only a spectator, we shall not write the spin component explicitly. As in the two-body case, the many-body wave function can be normalized in such a way that for $r_{0}$ $\ll\left|\vec{x}_{i}-\vec{y}_{j}\right| \lesssim a_{s}, k_{F}^{-1}$,

$$
\begin{array}{r}
\lim _{r_{0} \ll\left|\vec{x}_{i}-\vec{y}_{j}\right| \lesssim a_{s}, k_{F}^{-1}} \Psi\left(\vec{x}_{1}, \vec{x}_{2}, \ldots, \vec{y}_{1}, \vec{y}_{2}, \ldots\right) \\
=\frac{1}{\sqrt{4 \pi}} \frac{1}{\left|\vec{x}_{i}-\vec{y}_{j}\right|}\left(1-\frac{\left|\vec{x}_{i}-\vec{y}_{j}\right|}{a_{s}}\right) \Psi^{\prime},
\end{array}
$$

where $\Psi^{\prime}$ is a function of variables other than $\vec{x}_{i}$ and $\vec{y}_{j}$. We say that the many-body system is on resonance if $a_{s} \rightarrow \infty . a_{s}$ should be regarded as a parameter of the theory that can be tuned in the experiments by the external magnetic field. $\mathrm{Nu}$ merically, the value of $a_{s}$ as defined in Eq. (A7) must be essentially equal to that in the two-body case in the same magnetic field, as the discussions in Sec. II would imply.

To put our system in a finite volume such that the density of particles $n=N / \Omega$ is kept constant, we shall introduce the characteristic function of the volume $\Omega$,

$$
\chi_{\Omega}(\vec{x})=1 \quad \text { if } \vec{x} \in \Omega, \quad \text { otherwise zero. }
$$

Then we can enforce the condition of constant density through an external one-body potential $U(\vec{x})$,

$$
U(\vec{x})=U_{0}\left[1-\chi_{\Omega}(\vec{x})\right],
$$

where $U_{0}$ is a large constant representing the hard wall such that the many-body wave function vanishes outside the region $\Omega$ and on the boundary of $\Omega$, denoted by $\partial \Omega$,

$$
\left.\Psi(\vec{x}, \vec{y})\right|_{\partial \Omega}=0 .
$$

$\Psi(\vec{x}, \vec{y})$ is short-hand notation for $\Psi\left(\vec{x}_{1}, \vec{x}_{2}, \ldots, \vec{y}_{1}, \vec{y}_{2}, \ldots\right)$. The time-independent Schrödinger equation takes the form

$$
\begin{gathered}
\sum_{i}\left(-\frac{\hbar^{2}}{2 m} \nabla_{\vec{x}_{i}}-\frac{\hbar^{2}}{2 m} \nabla_{\vec{y}_{i}}+U\left(\vec{x}_{i}\right)+U\left(\vec{y}_{j}\right)\right) \Psi(\vec{x}, \vec{y}) \\
+\frac{1}{2} \sum_{i, j} V_{\lambda}\left(\vec{x}_{i}-\vec{y}_{j}\right) \Psi(\vec{x}, \vec{y})=E_{\lambda} \Psi(\vec{x}, \vec{y}) .
\end{gathered}
$$

In writing the above equation, we have neglected the interaction potential between parallel spins, corresponding to the Fock energy in a many-body system. This is certainly negligible as compared with the Hartree term between antiparallel spins, which has been incorporated in the above expression. We now follow the same recipe developed for the two-body case. We write another equation corresponding to $\lambda=\lambda_{0}$, i.e., corresponding to $a_{s} \rightarrow \infty$ at resonance. Let us also denote the corresponding energy by $E_{0}$ and the wave function by $\Psi_{0}(\vec{x}, \vec{y})$. Then by multiplying each equation with $\Psi_{\lambda}$ or $\Psi_{0}$, respectively, and subtracting against each other, we find

$$
E_{\lambda}-E_{0}=\frac{1}{2} \sum_{i, j} \frac{\int d \vec{x} d \vec{y} \delta V_{\lambda}\left(\vec{x}_{i}-\vec{y}_{j}\right) \Psi_{\lambda} \Psi_{0}}{\int d \vec{x} d \vec{y} \Psi_{\lambda} \Psi_{0}},
$$

where $\delta V_{\lambda}\left(\vec{x}_{i}-\vec{y}_{j}\right)=V_{\lambda}\left(\vec{x}_{i}-\vec{y}_{j}\right)-V_{\lambda_{c}}\left(\vec{x}_{i}-\vec{y}_{j}\right)$. We have used Green's theorem and the fact that the wave function vanishes at the boundary of the volume $\Omega$. In differential form, we have 


$$
\frac{d E_{\lambda}}{d \lambda}=\frac{1}{2} \sum_{i, j} \frac{\int d \vec{x} d \vec{y} \frac{\partial V_{\lambda}\left(\vec{x}_{i}-\vec{y}_{j}\right)}{\partial \lambda} \Psi_{\lambda} \Psi_{0}}{\int d \vec{x} d \vec{y} \Psi_{\lambda} \Psi_{0}} .
$$

Now, $\lambda$ determines the scattering length $a_{s}(\lambda)$ and thus if we use $\xi=-\left(k_{F} a_{s}\right)^{-1}$, we find, by using Eq. (13),

$$
\begin{aligned}
\frac{d E(\xi)}{d \xi} & =-\frac{d \lambda}{d a_{s}^{-1}} \frac{k_{F}}{2} \sum_{i, j} \frac{\int d \vec{x} d \vec{y} \frac{\partial V_{\lambda}\left(\vec{x}_{i}-\vec{y}_{j}\right)}{\partial \lambda}\left|\Psi_{0}\right|^{2}}{\int d \vec{x} d \vec{y} \Psi_{\lambda} \Psi_{0}} \\
& =-\frac{\hbar^{2}}{2 m} k_{F} \frac{\sum_{i, j} \int d \vec{x} d \vec{y} \frac{\partial V_{\lambda}}{\partial \lambda}\left|\Psi_{0}\right|^{2}}{\int_{0}^{\infty} d r \frac{\partial V_{\lambda}}{\partial \lambda}\left|\chi_{0}(r)\right|^{2}} \frac{1}{\int d \vec{x} d \vec{y} \Psi_{\lambda} \Psi_{0}} .
\end{aligned}
$$

Notice the resemblance of Eq. (A14) to Eq. (16). In fact, we have merely managed to express the function $h(\xi)$ in terms of the many-body wave function. We have replaced $\Psi_{\lambda}$ with $\Psi_{0}$ in the numerator since $\frac{\partial V_{\lambda}}{\partial \lambda}$ is a short-range function. The integral involving $\frac{\partial V_{\lambda}}{\partial \lambda}$ in the numerator only picks up the short-range contribution from the probability distribution function $\left|\Psi_{0}(\vec{x}, \vec{y})\right|^{2}$, and thus apart from a normalization constant that is finite, it cancels the factor $\int_{0}^{\infty} d r \frac{\partial V_{\lambda}}{\partial \lambda}\left|\chi_{0}(r)\right|^{2}$ in the denominator. Thus, all the sensitive short-range dependence has disappeared in the above expression and we are left with quantities that are independent of short-range complications. Note now that the factor $\int d \vec{x} d \vec{y} \Psi_{\lambda} \Psi_{0}$ approaches a constant as $\xi \rightarrow 0$ since it is merely the normalization factor for the wave function $\Psi_{0}$. This is why, from a many-body wavefunction point of view, the definition of the function $h(\xi, \tau)$ is universal to the dilute Fermi gas system, irrespective of its short-range potential. The complicated expression on the right-hand side of Eq. (A14) reduces to a simple combination of normalization constants and thus remains well-defined for $a_{s} \rightarrow \infty$. We have thus established the linear dependence of the energy on $\xi$ around resonance.

Finally, the extension of the above argument to finite temperature is straightforward. In the case of thermal equilibrium, we consider instead a distribution of eigenstates $|n\rangle$ with energy $E_{n}$, each occurring with probability given by the usual Boltzmann factor $e^{-E_{n} / k_{B} T}$. One can repeat, word by word, the above derivation, and thus we can conclude that at finite temperature, the $\xi$ dependence of energy away from resonance is linear. Notice that we have to fix the relative occupation of each single-particle state, which is equivalent to fixing the entropy, in order for the argument to go through. This is, of course, the usual adiabatic process. Here we also assume that the temperature is quite low so that its effects on the short-range wave function are irrelevant. The argument can be extended also to a nonequilibrium situation in which the probability of state $|n\rangle$ is given by $p_{n}, \Sigma p_{n}=1$. However, it is practically useless since the characterization of the system as "away from the resonance" is ambiguous and one is not likely to obtain any useful conclusions from the argument.
[1] I. Bloch, J. Dalibard, and W. Zwerger, Rev. Mod. Phys. 80, 885 (2008).

[2] S. Giorgini, L. P. Pitaevskii, and S. Stringari, Rev. Mod. Phys. 80, 1215 (2008).

[3] W. Ketterle and M. W. Zwierlein, Ultracold Fermi Gases, Proceedings of the International School of Physics "Enrico Fermi," Course CLXIV, Varenna, 2006, edited by M. Inguscio, W. Ketterle, and C. Salomon (IOS Press, Amsterdam, 2008).

[4] M. Randeria, in Bose-Einstein Condensation, edited by A. Griffin, D. W. Snokes, and S. Stringari (Cambridge University Press, Cambridge, UK, 1995).

[5] T. Köhler, K. Góral, and Paul S. Julienne, Rev. Mod. Phys. 78, 1311 (2006).

[6] The expression for $\delta_{c}$ as given in Ref. [7] is in terms of the parameter $\kappa$ defined by Eq. (4.A.17) in the cited reference. To obtain the expression in the text, we only need to notice that when expanding the detuning around resonance, we find, $\Delta B$ $=\kappa\left(\frac{\partial \delta}{\partial B}\right)^{-1}=\kappa \Delta \mu^{-1}$. Then by using Eq. (4.A.25), we obtain the result in the text. Note that we have ignored the magnetic field dependence of $\kappa$. See Ref. [7], pp. $163 \mathrm{ff}$.

[7] A. J. Leggett, Quantum Liquids (Oxford University Press, Oxford, 2006).

[8] D. M. Eagles, Phys. Rev. 186, 456 (1969).

[9] A. J. Leggett, in Modern Trends in the Theory of Condensed
Matter, Proceedings of the XVIth Karpacz. Winter School of Theoretical Physics, Karpacz, Poland, 1980 (Springer-Verlag, Berlin, 1980), pp. 13-27.

[10] P. Nozières and S. Schmitt-Rink, J. Low Temp. Phys. 59, 195 (1985).

[11] R. Haussmann, W. Rantner, S. Cerrito, and W. Zwerger, Phys. Rev. A 75, 023610 (2007).

[12] J. Carlson, S.-Y. Chang, V. R. Pandharipande, and K. E. Schmidt, Phys. Rev. Lett. 91, 050401 (2003).

[13] G. E. Astrakharchik, J. Boronat, J. Casulleras, and S. Giorgini, Phys. Rev. Lett. 93, 200404 (2004).

[14] S. Y. Chang and V. R. Pandharipande, Phys. Rev. Lett. 95, 080402 (2005).

[15] Evgeni Burovski, Nikolay Prokof'ev, Boris Svistunov, and Matthias Troyer, Phys. Rev. Lett. 96, 160402 (2006); see also 97, 239902(E) (2006).

[16] Aurel Bulgac, Joaquín E. Drut, and Piotr Magierski, Phys. Rev. Lett. 99, 120401 (2007).

[17] Vamsi K. Akkineni, D. M. Ceperley, and Nandini Trivedi, Phys. Rev. B 76, 165116 (2007).

[18] Alexandros Gezerlis and J. Carlson, Phys. Rev. C 77, 032801(R) (2008).

[19] G. B. Partridge, K. E. Strecker, R. I. Kamar, M. W. Jack, and R. G. Hulet, Phys. Rev. Lett. 95, 020404 (2005). 
[20] Q. Chen, J. Stajic, S. Tan, and K. Levin, Phys. Rep. 412, 1 (2005).

[21] M. Holland, S. J. J. M. F. Kokkelmans, M. L. Chiofalo, and R. Walser, Phys. Rev. Lett. 87, 120406 (2001).

[22] E. Timmermans, K. Furuya, P. W. Milonni, and A. K. Kerman, Phys. Lett. A 285, 228 (2001).

[23] T. L. Ho, Phys. Rev. Lett. 92, 090402 (2004).

[24] Predrag Nikolić and Subir Sachdev, Phys. Rev. A 75, 033608 (2007).

[25] M. Y. Veillette, D. E. Sheehy, and L. Radzihovsky, Phys. Rev. A 75, 043614 (2007).

[26] Y. Nishida and D. T. Son, Phys. Rev. A 75, 063617 (2007).

[27] Jiunn-Wei Chen and E. Nakano, Phys. Rev. A 75, 043620 (2007).

[28] A. A. Abrikosov, L. P. Gor'kov, and I. E. Dzyaloshinski, Methods of Quantum Field Theory in Statistical Physics (Dover Publication, New York, 1975).

[29] K. Huang and C. N. Yang, Phys. Rev. 105, 767 (1957).

[30] T. D. Lee and C. N. Yang, Phys. Rev. 105, 1119 (1957).

[31] V. Gurarie and L. Radzihovisky, Ann. Phys. (N.Y.) 322, 2 (2007).

[32] Shina Tan, Ann. Phys. (N.Y.) 323, 2952 (2008). We thank Eric Braaten and Lucas Platter for drawing our attentions to Tan's paper.

[33] E. Braaten and L. Platter, Phys. Rev. Lett. 100, 205301 (2008).

[34] Shina Tan, Ann. Phys. (N.Y.) 323, 2971 (2008).

[35] Shina Tan, Ann. Phys. (N.Y.) 323, 2987 (2008).
[36] C. Chin, M. Bartenstein, A. Altmeyer, S. Riedl, S. Jochim, J. Denschlag, and R. Grimm, Science 305, 1128 (2004).

[37] A. J. Leggett, Phys. Rev. Lett. 29, 1227 (1972).

[38] M. J. Leskinen, V. Apaja, J. Kajala, and P. Törmä, Phys. Rev. A 78, 023602 (2008).

[39] J. Kinnunen, M. Rodriguez, and P. Törmä, Science 305, 1131 (2004).

[40] Y. Ohashi and A. Griffin, Phys. Rev. A 72, 013601 (2005).

[41] Y. He, Q. Chen, and K. Levin, Phys. Rev. A 72, 011602(R) (2005).

[42] Z. Yu and G. Baym, Phys. Rev. A 73, 063601 (2006).

[43] G. Baym, C. J. Pethick, Z. Yu, and M. W. Zwierlein, Phys. Rev. Lett. 99, 190407 (2007).

[44] M. Punk and W. Zwerger, Phys. Rev. Lett. 99, 170404 (2007).

[45] A. Perali, P. Pieri, and G. C. Strinati, Phys. Rev. Lett. 100, 010402 (2008).

[46] S. Z. Zhang and A. J. Leggett, Phys. Rev. A 77, 033614 (2008).

[47] S. Basu and E. J. Mueller, Phys. Rev. Lett. 101, 060405 (2008).

[48] Qijin Chen and K. Levin, Phys. Rev. Lett. 95, 260406 (2005).

[49] M. W. J. Romans and H. T. C. Stoof, Phys. Rev. Lett. 95, 260407 (2005)

[50] J. Javanainen, M. Koštrun, M. Mackie, and A. Carmichael, Phys. Rev. Lett. 95, 110408 (2005).

[51] F. Werner, L. Tarruell, and Y. Castin, e-print arXiv:0807.0078. 